\title{
Application of the TRUFAS detection algorithm to the first two runs of CoRoT
}

\author{
Clara Régulo $^{1,2}$, Jose M. Almenara ${ }^{1}$ and Hans J. Deeg ${ }^{1}$ \\ ${ }^{1}$ Instituto de Astrofísica de Canarias, \\ 38205, La Laguna, Tenerife, Spain \\ email: [crr; jmav; hdeeg] @iac.es \\ ${ }^{2}$ Dept. de Astrofísica, Universidad de La Laguna, \\ 38206, La Laguna, Tenerife, Spain
}

\begin{abstract}
.
TRUFAS is a wavelet-based algorithm developed for the rapid detection of planetary transits in the frame of the COROT space mission. We present the application of this algorithm to the first two observing fields of CoRoT data. In these, CoRoT has observed a total of about 20000 stars. The first CoRoT observing run, IRa01, covers 2 months, February and March 2007, followed by the 5-months long run LRc01. TRUFAS is a very fast algorithm delivering reliable detections. Here we show the results when TRUFAS was applied to these first two sets of data. In the first run, IRa01, TRUFAS found 10 planet candidates and 143 eclipsing binaries and in the LRc01 10 planet candidates and 124 binaries, with a processing that lasted only one night.
\end{abstract}

\section{Introduction}

The TRUFAS algorithm was developed for the analysis of CoRoT data (Baglin et al. 2007). It was tested with a set of 999 simulated stellar light curves, created for the comparison of several detection algorithm within the "CoRoT blind test" (Moutou et al. 2005). The results obtained in this test were quite encouraging, because TRUFAS worked very fast and as well as the best of the other algorithms against which it was tested (Régulo et al. 2007).

In this work, TRUFAS is applied to the first two runs of real data obtained with CoRoT.

\section{Methodology}

The input for the planetary transits detection algorithm TRUFAS is the photometric light curve of the star, obtained from CoRoT (Fig. 1). The only step needed before starting the algorithm is a filter. The filter is implemented in the Fourier domain as a high pass filter, where the transition zone is automatically adjusted for each light curve according to its level of noise. This filtered light curve (Fig. 2), is introduced in the TRUFAS algorithm that is divided in five steps.

The first step (Fig. 3) is a Continuous Wavelet Transformation (CWT) of the filtered data, to select the best scale to look for the transit. CWT maps the correlation coefficients between the signal and the selected wavelet function (the Paul function). The scale with the higher coefficients is the best one to look for the transit. The selection of the scale in the CWT is the second step of TRUFAS (Fig. 4).

The third step is to obtain the power spectrum of the square of the selected scale (FFT) in order to have a set of equally spaced peaks with the first one at zero frequency 


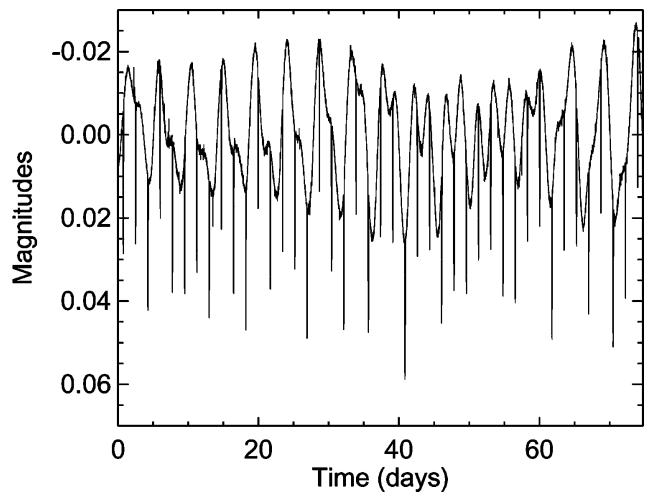

Figure 1. Photometric light curve from CoRot (input).

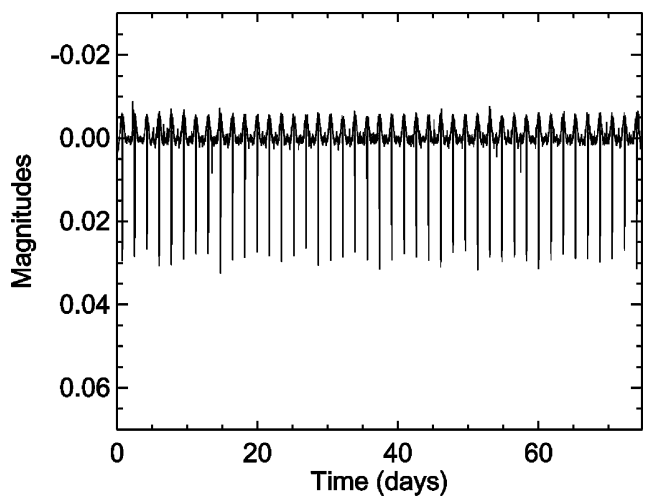

Figure 2. Filtered light curve with a Fourier Filter (pre-processing the data).

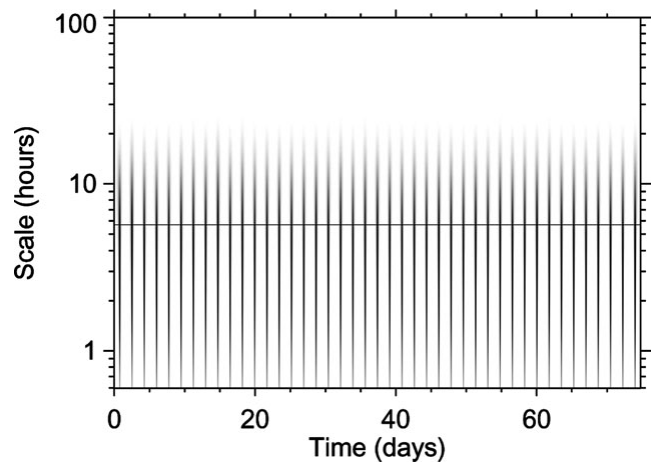

Figure 3. Continuous wavelet transform. The horizontal line indicates the scale to be selected. First Step of TRUFAS.

(Fig. 5). In the FFT, the spacing among peaks is found. These spacing will be $T^{-1}, T$ being the period of the transit.

The search for the spacing in the FFT is done iteratively trying a range of possible values that covers from 1 day to 60 days in steps of $512 \mathrm{~s}$. To evaluate the significance of the peaks found, the procedure is repeated 50 times changing slightly the length of the selected scale to avoid binning effects. The coincidences of periods among peaks found 


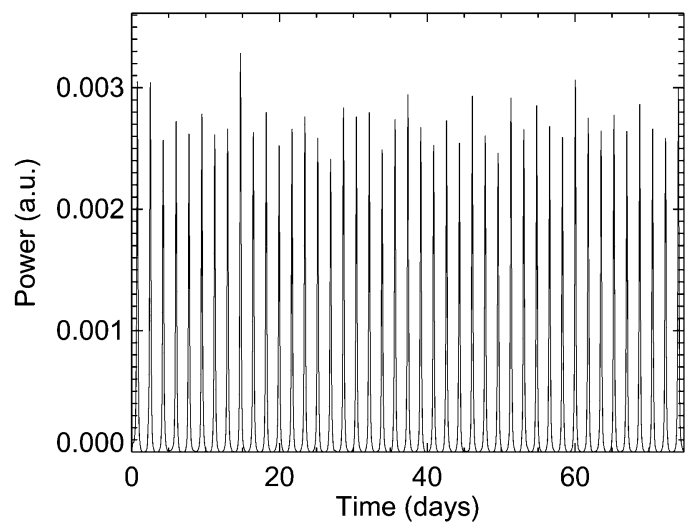

Figure 4. Selected scale, that corresponds with the horizontal line plots in Fig. 3. Second Step of TRUFAS.

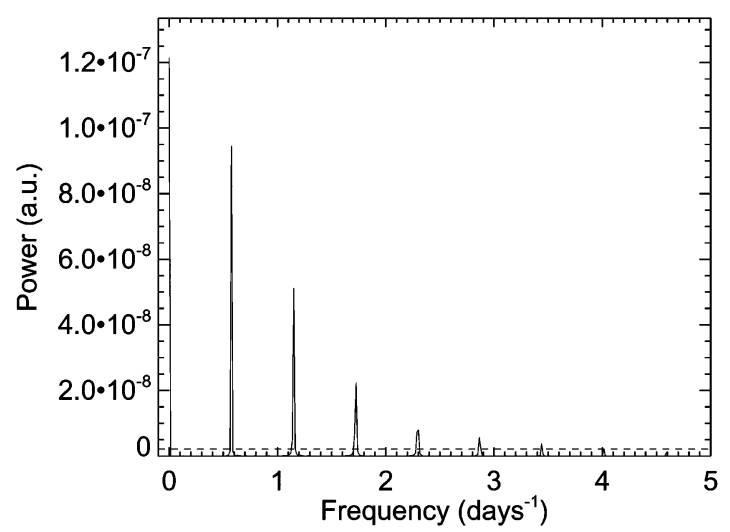

Figure 5. FFT of the selected scale. Third Step of TRUFAS.

in the 50 trials is registered. The fourth step is to count these coincidences (Fig. 6). The period with more coincidences is the one chosen as the period of the transit.

The last and fifth step of TRUFAS is done when all the light curves have been processed with the four previous steps, and it is the step where we select which star has a real transit among the results obtained. In this step (Fig. 7) a histogram from all the analysed light curves indicates the coincidences of the detected periods across the 50 trials. This histogram is used to select the number of coincidences above which the detection is considered as a real detection.

TRUFAS ends at this step with detected transits. These detected transits are then analysed for classification as binary systems, if the light curve shows primary and secondary eclipses of different depths, or as planet candidates, if the transits are of the same depth. This classification is done in an automatic way using hypothesis testing to take decisions (Almenara et al. 2008).

\section{Results}

The application of TRUFAS to real data works pretty well, although real data show some features quite different from the simulated data for which the algorithm had been optimized. 


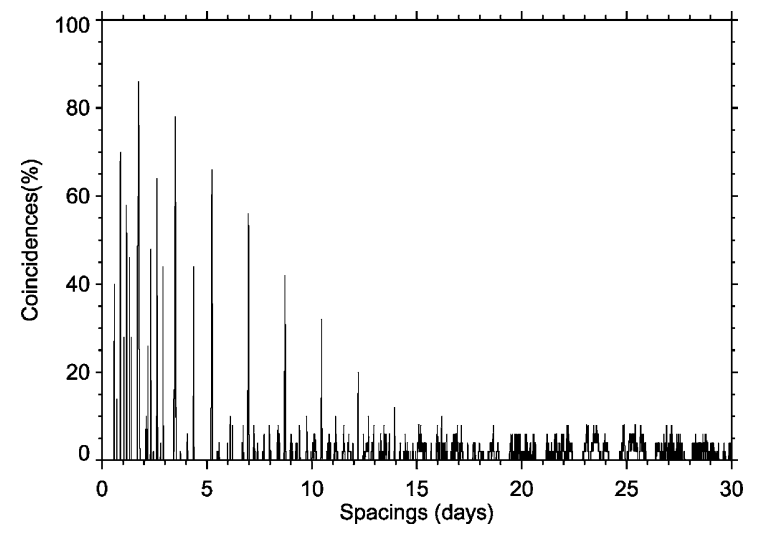

Figure 6. Coincidences found in the search for the possible transit period. The highest peak corresponds to the period of the transit present in the data. The other peaks are multiples and submultiples of the period present in the data. Fourth Step of TRUFAS.

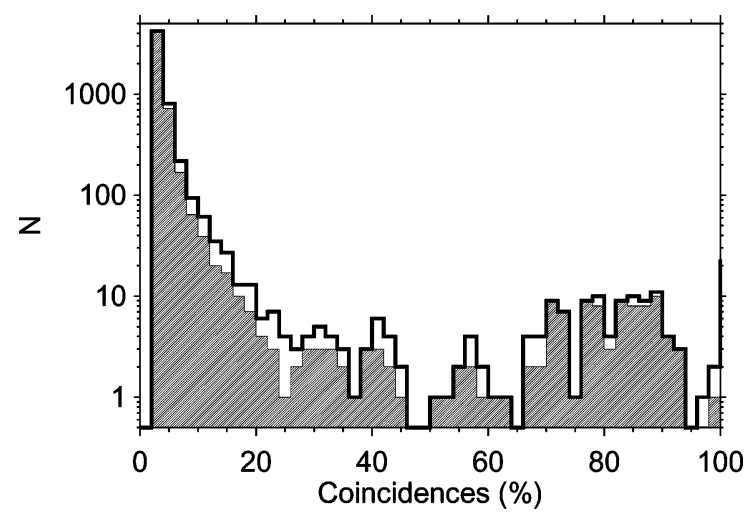

Figure 7. Histogram of the coincidences for all the analysed light curves. Fifth Step of TRUFAS.

In only one night, TRUFAS managed to analyse each set of data. The first run, IRa01, with 9872 stars, covers 2 months, February and March 2007. From this set, 143 binary systems were detected as well as 10 planet candidates. From the second run, LRc01, with 11408 stars measured during 5 months, from April to August 2007, 124 binary systems were detected and again 10 planet candidates.

\section{References}

Almenara, J. M \& Deeg, H. J. 2008, in press

Baglin, A. \& CoRoT Team 2007, AIPC, 895, 201

Moutou, C., Pont, F., Barge, P., et al. 2005, A\&SA, 437, 355

Régulo, C., Almenara, J.M., Alonso, R., Deeg, H., \& Roca Cortés, T. 2007, A\& A, 467, 1325 